\title{
Changes in the distribution pattern of Claudin tight junction proteins during the progression of mouse skin tumorigenesis Azadeh Arabzadeh ${ }^{\dagger 1,2}$, Tammy-Claire Troy ${ }^{\dagger 1}$ and Kursad Turksen*1,2,3,4
}

Address: ${ }^{1}$ Ottawa Health Research Institute, Ottawa, Ontario, K1Y 4E9 Canada, ${ }^{2}$ Department of Cellular and Molecular Medicine, Faculty of Medicine, University of Ottawa, Ottawa, Ontario, K1H 8M5 Canada, ${ }^{3}$ Department of Medicine, Divisions of Dermatology and Endocrinology, Faculty of Medicine, University of Ottawa, Ottawa, Ontario, K1H 8M5 Canada and ${ }^{4}$ Department of Obstetrics, Gynaecology, Division of Reproductive Endocrinology, Faculty of Medicine, University of Ottawa, Ottawa, Ontario, K1H 8M5 Canada

Email: Azadeh Arabzadeh - aarabzadeh@ohri.ca; Tammy-Claire Troy - ttroy@ohri.ca; Kursad Turksen* - kturksen@ohri.ca

* Corresponding author †Equal contributors

Published: 18 October 2007

BMC Cancer 2007, 7:196 doi:10.1186/147|-2407-7-196
Received: 23 May 2007

Accepted: 18 October 2007

This article is available from: http://www.biomedcentral.com//47/-2407/7//96

(c) 2007 Arabzadeh et al; licensee BioMed Central Ltd.

This is an Open Access article distributed under the terms of the Creative Commons Attribution License (http://creativecommons.org/licenses/by/2.0), which permits unrestricted use, distribution, and reproduction in any medium, provided the original work is properly cited.

\begin{abstract}
Background: Despite the fact that morphological and physiological observations suggest that the tight junction (TJ)-based permeability barrier is modified/disrupted in tumorigenesis, the role of members of the Claudin (Cldn) family of TJ proteins is not well-understood. Using a well-established two-stage chemical carcinogenesis model, we investigated the temporal and spatial changes in expression of those Cldns that we have previously demonstrated to be important in epidermal differentiation and the formation of the epidermal permeability barrier, i.e., Cldn I, Cldn6, Cldn II, Cldn 12 and Cldn I8.
\end{abstract}

Methods: The lower dorsal backskin of mice was treated topically with 7, I2-dimethylbenz(a)anthracene (DMBA; $0.25 \mathrm{mg} / \mathrm{ml}$ in acetone) and following a I0-day incubation period, 12-O-tetradecanoyl-phorbol- I3-acetate (TPA; $25 \mu \mathrm{g} / \mathrm{ml}$ in acetone) was applied three times a week to the same area. Backskin samples were dissected 2, 4, 6, 8 and 12 weeks after the initiation of the experimental protocol and immunohistochemistry was performed on sections using antibodies against the following: Cldn I, Cldn6, Cldn I I, Cldn I2, Cldn I8, Ki67 and CD3.

Results: Our data indicate that along with the changes in epidermal cell morphology and differentiation that occur during tumor formation, there is a dramatic change in Cldn distribution consistent with cell polarity and barrier selectivity changes. Specifically, in the early stages of DMBA/TPA treatment, the suprabasal-specific Cldns occupy an expanded zone of expression corresponding to an increased number of suprabasal epidermal cell layers. As tumorigenesis progressed, the number of suprabasal epidermal layers positive for Cldn6, Cldn II, Cldn 12 and Cldn 18 was reduced, especially in the lower strata of the expanded suprabasal zone. In addition, a variably reduced cell membrane association of those differentiation-specific Cldns was observed, especially within the infiltrating epidermal structures. In contrast, Cldn I (which is normally expressed in all the living layers of the epidermis) remained restricted to the cell membrane throughout the tumorigenesis protocol. However commencing 2 weeks after treatment there was a marked decrease in the number of Cldn I-positive basal cells, and the zone of CldnI-null epidermal cells was expanded up into the lower stratified epidermis throughout the progression of DMBA/TPA treatment. In addition, there was no Cldn I localization in the infiltrating epidermal structures of the tumorigenic epidermis.

Conclusion: This is the first demonstration of the changes in Cldn expression in the progression of DMBA/TPAinduced skin tumors; however further investigation into the molecular mechanisms regulating the observed changes in barrier selectivity during tumorigenesis is required. 


\section{Background}

Disruption of epithelial cell polarity and cell-cell junctions with concomitant changes in the expression of junctional proteins during primary tumor formation is considered to be a hallmark of cancer cell invasion and metastasis [1]. Amongst the junctional complexes, the role in tumor formation of specific tight junction (TJ) proteins essential for cell polarity and the formation and maintenance of heterogeneous permeability barriers is not well understood. In vivo studies have demonstrated that the epidermis is characterized by a defined differentiation-dependent expression of Claudins (Cldns), a family of tetraspan membrane proteins that comprise a major component of TJ fibrils essential to the structure and function of TJs [2-6]. Recent studies have also shown that changes in the distribution pattern of diverse Cldns may contribute to changes observed in cell permeability [710]. However, no systematic analysis of the expression and/or localization of various Cldns during skin tumorigenesis is yet available.

Understanding the molecular basis of skin tumor development has been greatly facilitated by the use of animal model systems in which tumor development can be carefully controlled [11]. For example, the classical mouse two-stage carcinogenesis model provides an excellent system in which to study the stages and molecular mechanisms involved in squamous cell carcinoma [12]. The chemical carcinogenesis process can be divided into three distinct phases: initiation, promotion and progression. Initiation results from exposure to a mutagenic carcinogen, followed by the application of a promoter to alter gene expression and increase cell proliferation to ultimately result in tumor formation. Therefore, we used this model to elucidate the changes occurring in Cldn expression during the progression of epithelial tumors.

\section{Methods}

\section{DMBA/TPA treatment}

The coat on the dorsal side of one-month-old CD1 wild type mice was shaved one day prior to the initiation of the experimental protocol and mice were shaved once a week as required until the coat failed to re-grow. The lower dorsal backskin of mice was treated topically with 7,12dimethylbenz( $a$ )anthracene (DMBA; $0.25 \mathrm{mg} / \mathrm{ml}$ in acetone) and following a 10-day incubation period, 12-O-tetradecanoyl-phorbol-13-acetate (TPA; $25 \mu \mathrm{g} / \mathrm{ml}$ in acetone) was applied three times a week to the same area. Experimental results were highly reproducible in three independent assays, each comprising three mice per time point (treated vs. control) and a minimum of 2-3 biopsies per mouse. All animal studies were conducted according to the regulations of the Canadian Council on Animal Care.

\section{Sample Collection}

Backskin samples $\left(\sim 1 \mathrm{~cm}^{2}\right)$ were dissected from the lesion and/or tumor regions of the mid-dorsal backskin from DMBA/TPA-treated mice as well as their vehicle-treated controls; sampling was done 2, 4, 6, 8 and 12 weeks after the initiation of the experimental protocol. Since the fixation method routinely used in our laboratory is rather stringent (see below) and not appropriate for all antibodies, frozen sections were required for Cldn1, Ki67 and CD3 immunostaining [2]. All other immunolocalization and histology (Hematoxylin \& Eosin; H\&E) were performed on paraffin sections. For frozen sections: skin samples were embedded in HistoPrep ${ }^{\mathrm{TM}}$ and solidified in dry ice-chilled isopentane. Sections $(5 \mu \mathrm{m})$ on slides were warmed at room temperature for 3 minutes, and then fixed for 10 minutes in methanol at $-20^{\circ} \mathrm{C}$, followed by washing in PBS prior to immunostaining. For paraffin sections: skin samples were fixed for 12-16 hours in Bouin's fixative $(75 \%$ saturated picric acid, $20 \%$ formaldehyde and $5 \%$ glacial acetic acid) at room temperature, followed by ethanol dehydration $(30 \%, 50 \%, 70 \%, 95 \%, 100 \%)$, paraffin embedding and sectioning $(5 \mu \mathrm{m})$. Prior to H\&E and immunostaining, sections were dewaxed and rehydrated followed by antigen unmasking and washing steps [5].

\section{Immunohistochemistry}

Paraffin and frozen sections were blocked for non-specific antibody binding by a 30-minute room temperature incubation (10\% goat serum, $0.8 \%$ BSA, $1 \%$ gelatin in PBS) followed by several washes in wash buffer $(0.8 \%$ BSA, $1 \%$ gelatin in PBS). Primary antibodies appropriately diluted in incubation buffer ( $1 \%$ goat serum, $0.8 \%$ BSA, $1 \%$ gelatin in PBS) were applied for 1-2 hours at room temperature; antibodies against the following were used: Ki67 (1:25) (cat. \# ab833; abcam, Cambridge, MA), CD3 (1:100) (cat. \# 555273; BD Biosciences, Franklin Lakes, NJ), Cldn6 (1:50) (custom antibody generated from hen \#3677 against mouse Cldn6 sequence-CYSTSVPHSRGPSEYPTKNYV, Aves Labs, Inc., San Diego, CA), Cldn11 (1:50) (custom antibody generated from hen \#3680 against mouse Cldn11 sequence-CRKMDELGSK, Aves Labs, Inc.), Cldn12 (1:50) (custom antibody generated from hen \#5186 against mouse Cldn12 sequence-CZRKLRLITFNRNEKNLTIYT, Aves Labs, Inc.), Cldn18 (1:50) (custom antibody generated from rabbit \#A9953 against mouse Cldn 18 sequence-CRTEDDEQSHPTKYDYV, Open Biosystems, Huntsville AL) and Cldn1 (6:100) (cat. \#71-7800; Invitrogen, Burlington, Canada). Following incubation in wash buffer, secondary antibodies against rabbit, rat and chicken conjugated to FITC (Jackson ImmunoResearch Laboratories, West Grove, PA) were used at a 1:50 dilution in incubation buffer for 1 hour at room temperature. Images were acquired using a Zeiss Axioplan 2 brightfield/fluorescence capable microscope 
outfitted with an AxioCam camera and Axio Vision 2.05 software (Carl Zeiss Canada Ltd, Toronto, Canada) before processing with Adobe Photoshop version 7.0 (Adobe Systems, Inc., San Jose, CA). a
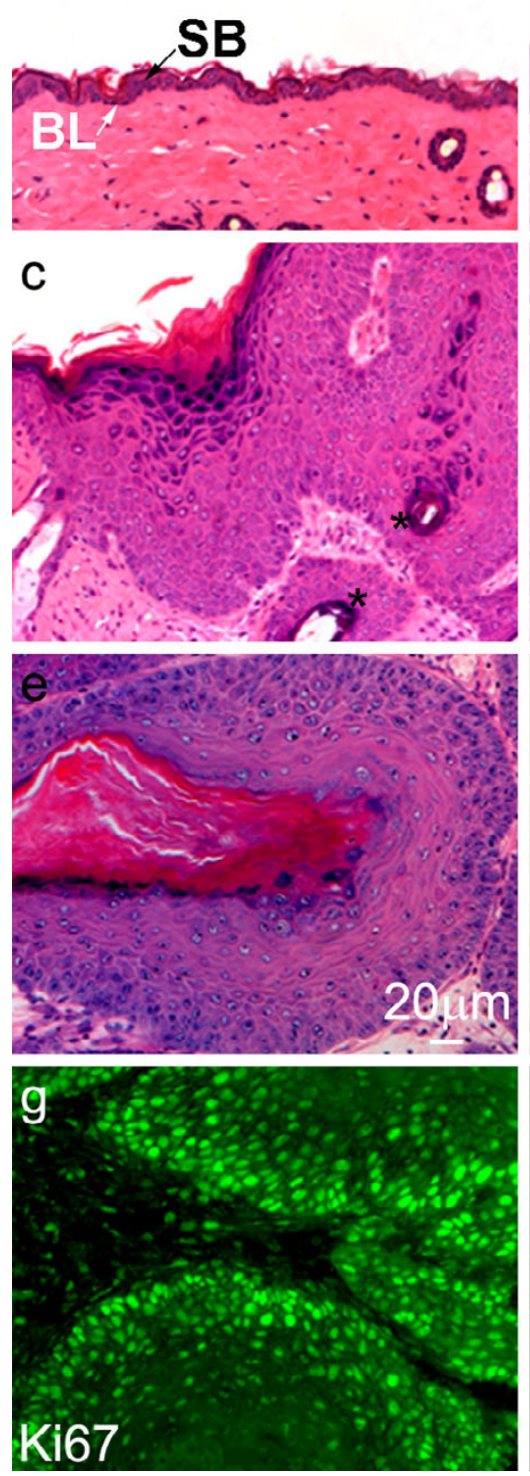

\section{Results and discussion}

\section{Characteristics of skin tumor progression}

When treated with vehicle (acetone) only for 12 weeks, the epidermis (Figure 1a) was morphologically reminiscent of the normal, untreated epidermis in age-matched samples (not shown). Initiation with DMBA followed by 2 to 12 weeks of TPA treatment caused the epidermis pro-

b
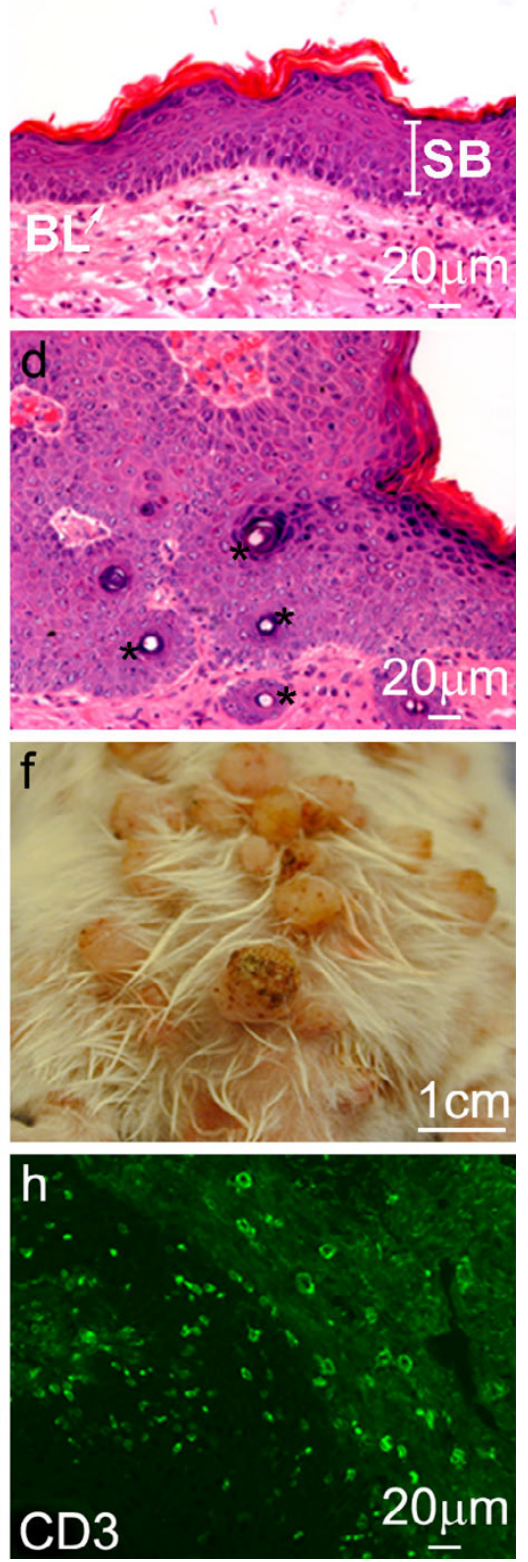

\section{Figure I}

Histological characteristics of skin tumor formation. H\&E staining of the vehicle-treated epidermis after 12 weeks is shown (a). The epidermis after 4 (b), 6 (c), 8 (d) and 12 (e) weeks of the classical two-stage chemical carcinogenesis protocol revealed striking abnormalities in epidermal architecture consistent with tumor formation, including the appearance of pseudohorn cysts (marked with stars). The dorsal skin of the mouse after 12 weeks of DMBA/TPA treatment is shown (f). Both Ki67-positive cells (g) and CD3-positive infiltrates (h) were evident throughout the experimental protocol ( 12 weeks is shown). BL: basal layer; SB: suprabasal compartment. 
gressively to display abnormalities in morphological architecture consistent with tumor formation, including a much thicker suprabasal compartment, an expanded stratum corneum and a more dispersed granular layer (Figure $1 b-e)$. In addition, characteristic invaginations of epidermal structures into the dermis were evident (Figure 1c-e), as were pseudohorn cysts (Figure 1c-d, marked with stars); a photograph depicting the dorsal side of the DMBA/TPA-treated mouse after 12 weeks is shown (Figure 1f). Throughout the experimental protocol, immunofluorescence confirmed the expression of Ki67 (Figure $1 \mathrm{~g}$ ), a nuclear protein expressed by proliferating cells during all phases of the cell cycle, and the T-cell receptor-associated CD3 complex (Figure 1h), indicative of the proliferative and immune infiltrative states of the treated epidermis. The expression and localization of keratins and epidermal terminal differentiation markers were also progressively disrupted as has been previously described (not shown) [13].

\section{Expression of the suprabasal Cldns in skin tumorigenesis}

We next analyzed the expression of those Cldns we have previously demonstrated to be important in epidermal differentiation and the formation of the epidermal permeability barrier $[2,4,5,14]$. The distribution of Cldn6, Cldn11, Cldn 12 and Cldn18 was indistinguishable in the normal (not shown) and vehicle-treated (Figure 2m-p) epidermis after 12 weeks, occupying the entire suprabasal compartment (in Figure 2 the basal layer is marked by a dotted line and the suprabasal compartment is indicated with a bracket). After 2 weeks of DMBA/TPA treatment (Figure 2a-d), on the other hand, the suprabasal compartment was expanded, with a corresponding expanded zone of Cldn expression in all the suprabasal layers. However, after 6 (Figure 2e-h), 8 (Figure 3a-d) and 12 (Figure 2i-l) weeks, the treated epidermis displayed a marked reduction in the number of suprabasal cell layers staining positively for Cldn6, Cldn11, Cldn 12 and Cldn18; the loss of staining was evident in the lower suprabasal zone (Figure 3; representative areas of Cldn-negative suprabasal cells are marked with stars). In addition there was an obvious but somewhat variable shift in the subcellular localization of all four of these Cldns away from the cell membrane that was especially evident in the lower suprabasal layers of the DMBA/TPA-treated epidermis after 8 weeks (Figure $3 \mathrm{a}-\mathrm{d}$, arrowheads illustrate representative areas where Cldn association is not restricted to cell membranes). Although all of the suprabasal-specific Cldns assayed showed some degree of non-membranous labeling after 8 weeks of treatment, this was especially true for Cldn 12 and Cldn 18 where the subcellular shift extended higher into the suprabasal zone than for Cldn6 and Cldn 11.

In the characteristic epidermal structures infiltrating the dermis of tumors after 8 weeks of treatment, the subcellu- lar translocalization of the suprabasal Cldns away from the cell membrane was even more apparent, with a nearly complete loss of membrane labeling (Figure $3 \mathrm{e}-\mathrm{h}$; the suprabasal compartment is marked with a double-ended arrow to demonstrate that the view encompasses only suprabasal cells and arrowheads point to representative areas of non-membranous Cldn labeling). In addition, there were many Cldn6-, Cldn11-, Cldn12- and Cldn18negative suprabasal cell layers evident (note that in Figure 3 , the stars highlight areas of Cldn-negative epidermal cells).

The expanded suprabasal Cldn expression compartment in early DMBA/TPA treatment, followed by the loss of Cldn6, Cldn11, Cldn12 and Cldn 18 in the lower strata of the suprabasal compartment as well as the reduced cell membrane association of those Cldns primarily associated with the stratifying/differentiating layers of the normal epidermis raises the question of whether such changes are obligatory for the promotional stage of skin tumor formation. The change in Cldn expression and localization may also impart new permeability properties to the affected and surrounding epidermal cells resulting in their hyperplastic conversion. Our results are consistent with the hypothesis of Daugherty et al. [15] that undifferentiated cells with poor barrier function exhibit a significant intracellular Cldn pool. In addition to transcriptional and translational regulation of expression levels, barrier function may be influenced by Cldn subcellular localization. In this context the observed changes in Cldn localization described in this study may be a result of multiple mechanisms including changes in Cldn phosphorylation. Although a number of Cldns, including the ones assessed in this study, have computer-predicted phosphorylation sites in the cytoplasmic tail domain, the functional significance of such sites has been demonstrated for only a few Cldns; e.g. Cldn3 where post-translational phosphorylation at threonine 192 has been shown to regulate TJ barrier function in ovarian cancer cells [16]. Another possibility is that there is increased endocytosis and/or an inhibition of a post-translational insertion into the ER resulting in Cldn retention in cytoplasmic vesicles. However, further investigation into the molecular mechanism(s) governing Cldn expression and localization in skin tumorigenesis is required.

\section{Changes in Cldn I expression through epidermal tumor progression}

Amongst the Cldns in the epidermis, Cldn 1 expression is unique and undergoes a maturation switch parallel to the acquisition of epidermal barrier function. In the developing epidermis, Cldn 1 expression is first restricted to the stratified layers at E15.5, and by E17.5 it occupies both the basal and suprabasal compartments [4]. This mature expression pattern is maintained throughout life under 

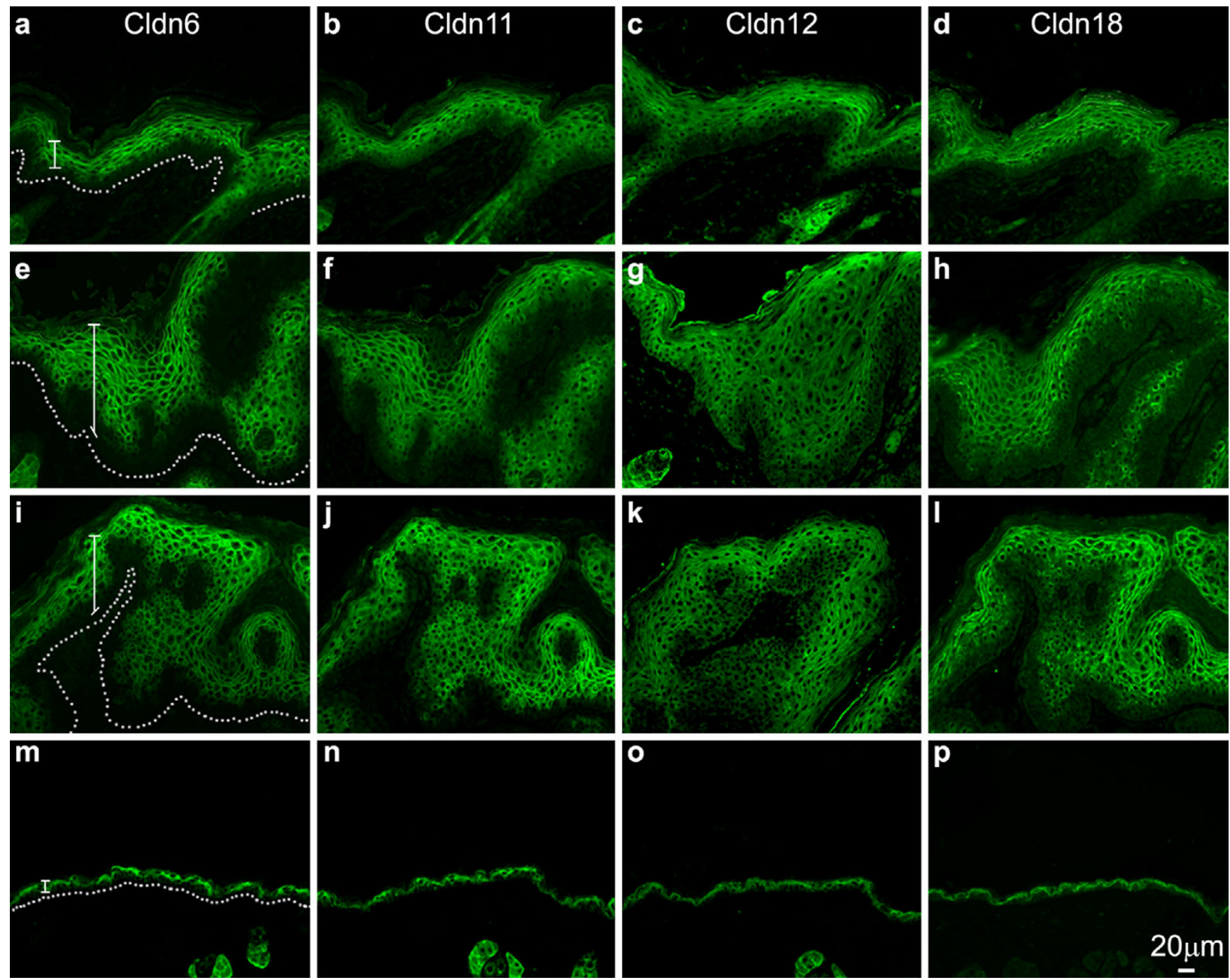

Figure 2

Suprabasal-specific Cldns in epidermal tumor progression. Cldn6, Cldn I I, Cldn I 2 and Cldn I 8 expression changes during epidermal tumorigenesis to occupy a gradually expanding zone of expression as observed after 2 (a-d), 6 (e-h) and I2 (i-I) weeks of the carcinogenesis protocol. While the localization of these suprabasal-specific Cldns shifted to varying degrees away from the cell membrane, there was a correspondingly varied reduction in the number of Cldn-positive epidermal cell layers in the lower strata of the epidermis after 6 and 12 weeks. The acetone-treated epidermis (after 12 weeks) is shown ( $\mathrm{m}$ - $\mathrm{p}$ ). A dotted line marks the base of the epidermal basal layer and the suprabasal zone is indicated with a bracket.

normal conditions and was not modified when the epidermis was exposed to vehicle over the duration of tumorigenesis experiments (Figure $4 \mathrm{a}$, the acetone-treated epidermis after 12 weeks is shown). However in response to DMBA/TPA treatment, Cldn1 lost its normal distribution pattern and adopted an expression like the immature pattern (Figure 4). Thus, 2 weeks after initiation of the carcinogenesis protocol, Cldn1-positive cells were decreased in the basal layer of the epidermis, while the entire suprabasal compartment maintained cell membrane-associated Cldn 1 expression (Figure 4b); by 4 weeks of treatment, the basal layer was essentially devoid of Cldn 1 (Figure $4 \mathrm{c})$. After 8 weeks of treatment, the zone of Cldn1-negative cells expanded upwards into the lower strata of the suprabasal compartment, with only sporadic presence of Cldn 1 protein remaining (Figure $4 \mathrm{~d}$ ), a phenomena more exaggerated after 12 weeks (Figure $4 \mathrm{e}$ ). However, and in contrast to the normal suprabasal-specific Cldns, the membranous localization of Cldn 1 was preserved throughout the 12 weeks of sampling in the upper strata of the suprabasal epidermis. Reminiscent of the reduced number of Cldn6, Cldn11, Cldn12 and Cldn18-positive 

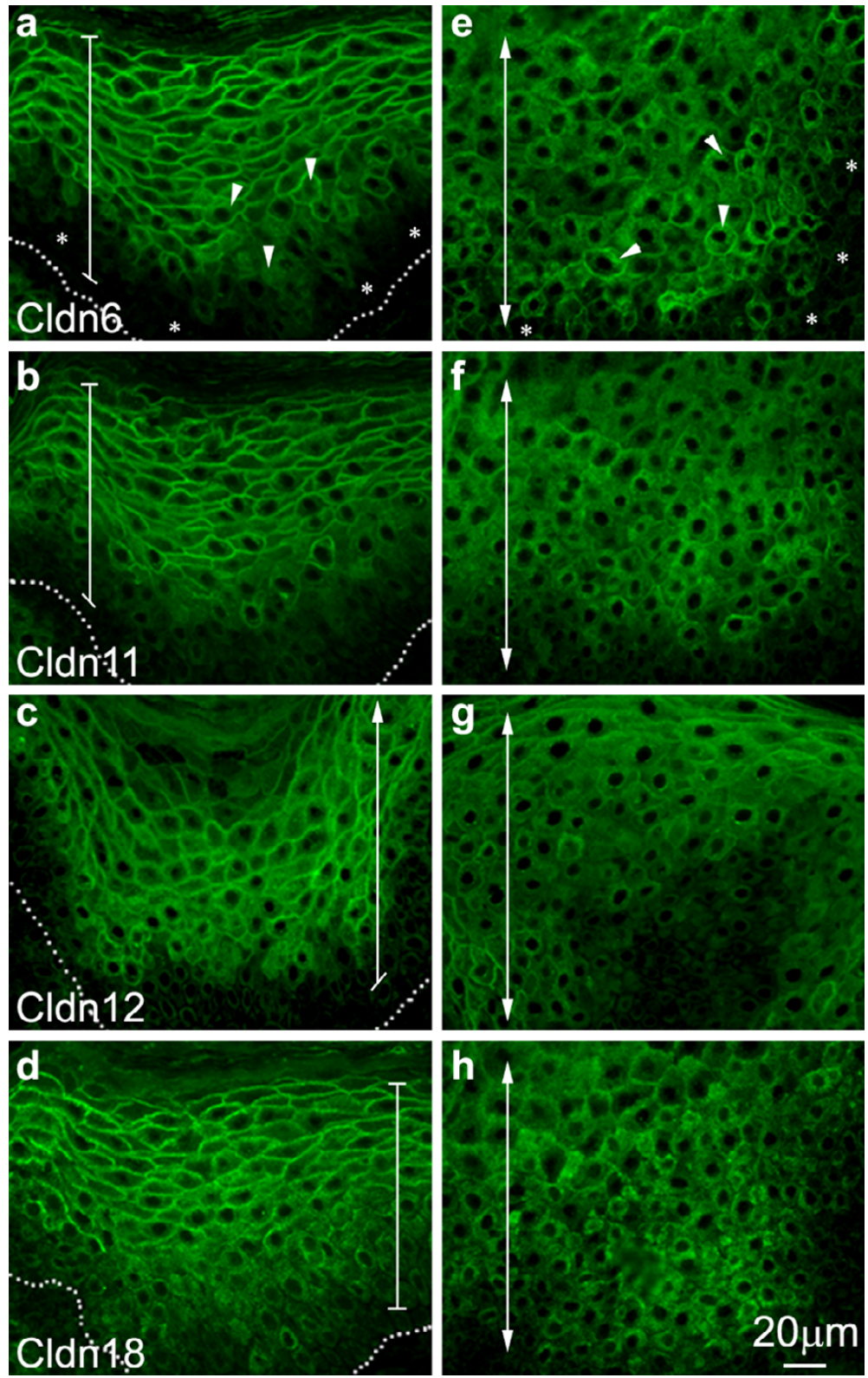

Figure 3

Differential Cldn localization in the tumorigenic epidermis. Higher magnification immunolocalization images of the epidermis (a-d) as compared to the characteristic epidermal structures infiltrating the dermis (e-h) better demonstrate the differences in Cldn localization 8 weeks after the initiation of the DMBA/TPA protocol. Membranous Cldn association was more prominent for each Cldn, albeit to varying degrees, in the upper strata of the epidermis; whereas in the lower strata and the epidermal invaginations Cldn localization was less membranous in nature and Cldn-null epidermal cells were more frequently observed. A dotted line marks the basal layer, and a bracket encompasses the epidermal suprabasal compartment in a-d. A double-ended arrow marks the suprabasal compartment in e-h to demonstrate that the entire panel represents suprabasal cells; arrowheads point to representative areas of non-membranous Cldn localization, and stars designate areas of Cldn-negative epidermal cells.

epithelial cell layers in the characteristic epidermal structures infiltrating the dermis, these areas were completely devoid of Cldn 1 expression (not shown).
Although the molecular mechanisms responsible for the reduced number of Cldn1-positive cells in epithelial tumorigenesis have not been defined, one possibility involves suppression by the Snail family of genes, which are 

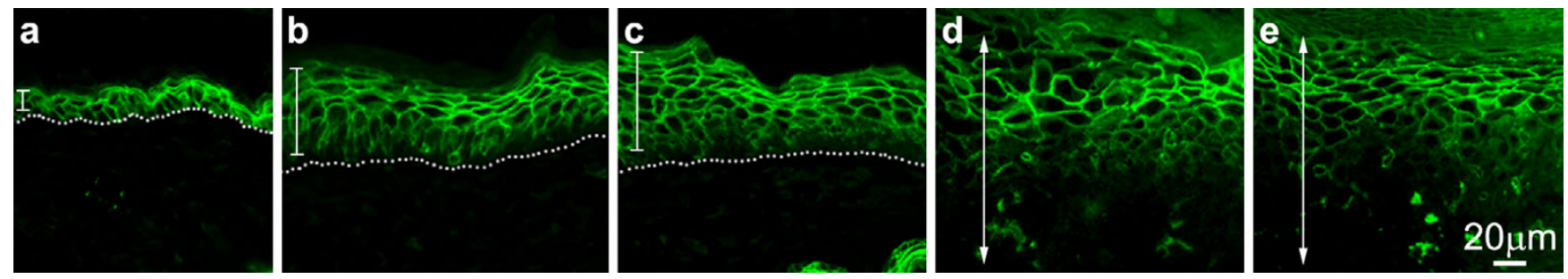

Figure 4

Changes in Cldn I expression in skin tumorigenesis. In the normal (not shown) and vehicle-treated (a, after I 2 weeks) epidermis, Cldn I is localized in the basal and suprabasal layers; however in response to the two-stage chemical carcinogenesis protocol, the number of Cldn I-positive epithelial cells was progressively reduced starting from the basal layer and moving upwards at 2 (b), 4 (c), 8 (d) and 12 (e) weeks. Although a distinctly membranous Cldn I association was maintained in the upper layers of the treated epidermis, as the number of Cldn I-negative epidermal cells in the lower epidermal layers increased, only sporadic Cldn I localization was evident (c-e). The epidermal basal layer is indicated by a dotted line, and the suprabasal compartment is marked with a bracket (a-c); note that the basal layer is out of view in panels $\mathbf{d}$ and $\mathbf{e}$; the entire view is therefore the suprabasal compartment and is marked with a double-ended arrow.

involved in numerous tumors both in vivo and in vitro [17]. In support of this notion, functional Snail-binding E-box motifs have been demonstrated to have a suppressive role on Cldn 1 expression in vitro $[18,19]$. Furthermore, a role for promoter methylation in the silencing of Cldn3, Cldn4 and Cldn7 has been observed in some tumors [20,21], suggesting that methylation could also play a role. Further studies are required to identify the mechanisms responsible for the changes observed in Cldn expression through epidermal tumor progression. However, our studies indicate that in this model the expression and distribution of Cldns change drastically and in a manner consistent with the loss of cell polarity and altered barrier selectivity concomitant with epidermal tumor formation.

\section{Abbreviations used}

Cldns: Claudins;

TJs: Tight junctions;

DMBA: 7,12-dimethylbenz(a)anthracene;

TPA: 12-O-tetradecanoyl-phorbol-13-acetate.

\section{Competing interests}

The author(s) declare that they have no competing interests.

\section{Authors' contributions}

AA's contribution entails the acquisition and presentation of data. TT's contribution involves the generation of reagents, the presentation of data as well as the drafting and revising of the manuscript. KT has made substantial intellectual contributions to the conception, design, analysis and interpretation of the data in addition to revising the manuscript for intellectual content and has given final approval of the version to be published. All authors have read and approved the final manuscript.

\section{Acknowledgements}

We sincerely appreciate Dr. Jane E. Aubin (University of Toronto) for her tremendous encouragement in every aspect of our research. We would also like to acknowledge Mrs. Adriana Gambarotta and Mr. Pierre Bradley, dedicated members of our animal care team; and we thank Zaida J. Ticas (MLT) (University of Ottawa) for preparing our histological sections. KT would like to thank Ingmar Bergman for Wild Strawberries and Michelangelo Antonioni for Blow-Up. This work was sponsored by a research grant from the Canadian Institutes of Health Research (MOP69087).

\section{References}

I. Mullin JM: Epithelial barriers, compartmentation, and cancer. SCi STKE 2004, 2004:pe2.

2. Arabzadeh A, Troy TC, Turksen K: Role of the Cldn6 cytoplasmic tail domain in membrane targeting and epidermal differentiation in vivo. Mol Cell Biol 2006, 26:5876-5887.

3. Furuse $M$, Hata $M$, Furuse $K$, Yoshida $Y$, Haratake $A$, Sugitani $Y$, Noda T, Kubo A, Tsukita S: Claudin-based tight junctions are crucial for the mammalian epidermal barrier: a lesson from claudinI-deficient mice. J Cell Biol 2002, I 56:1099-IIIII.

4. Troy TC, Li Y, O'Malley L, Turksen K: The temporal and spatial expression of Claudins in epidermal development and the accelerated program of epidermal differentiation in K14CaSR transgenic mice. Gene Expr Patterns 2007, 7:423-430.

5. Turksen K, Troy TC: Permeability barrier dysfunction in transgenic mice overexpressing claudin 6. Development 2002, 129:1775-1784.

6. Turksen K, Troy TC: Barriers built on claudins. J Cell Sci 2004, I I 7:2435-2447.

7. Furuse $M$, Tsukita $S$ : Claudins in occluding junctions of humans and flies. Trends Cell Biol 2006, 16:18I-188.

8. Koval M: Claudins--key pieces in the tight junction puzzle. Cell Commun Adhes 2006, 13:127-138.

9. Morin PJ: Claudin proteins in human cancer: promising new targets for diagnosis and therapy. Cancer Res 2005, 65:9603-9606.

10. Van Itallie CM, Anderson JM: Claudins and epithelial paracellular transport. Annu Rev Physiol 2006, 68:403-429.

II. Hirst GL, Balmain A: Forty years of cancer modelling in the mouse. Eur J Cancer 2004, 40:1974-1980. 
12. Yuspa SH: Overview of carcinogenesis: past, present and future. Carcinogenesis 2000, 21:34I-344.

13. Roop DR, Mehrel T, Krieg TM, Nakazawa H, Cheng CK, Yuspa SH: Keratin expression in mouse epidermal tumors. Carcinog Compr Surv 1989, I I:257-27I.

14. Troy TC, Rahbar R, Arabzadeh A, Cheung RM, Turksen K: Delayed epidermal permeability barrier formation and hair follicle aberrations in Inv-Cldn6 mice. Mech Dev 2005, 122:805-8I9.

15. Daugherty BL, Mateescu M, Patel AS, Wade K, Kimura S, Gonzales LW, Guttentag S, Ballard PL, Koval M: Developmental regulation of claudin localization by fetal alveolar epithelial cells. Am J Physiol Lung Cell Mol Physiol 2004, 287: LI 266-73.

16. D'Souza T, Agarwal R, Morin PJ: Phosphorylation of claudin-3 at threonine 192 by cAMP-dependent protein kinase regulates tight junction barrier function in ovarian cancer cells. J Biol Chem 2005, 280:26233-26240.

17. Barrallo-Gimeno A, Nieto MA: The Snail genes as inducers of cell movement and survival: implications in development and cancer. Development 2005, I32:3151-3161.

18. Martinez-Estrada OM, Culleres A, Soriano FX, Peinado $H$, Bolos $V$, Martinez FO, Reina M, Cano A, Fabre M, Vilaro S: The transcription factors Slug and Snail act as repressors of Claudin-I expression in epithelial cells. Biochem J 2006, 394:449-457.

19. Ohkubo T, Ozawa M: The transcription factor Snail downregulates the tight junction components independently of E-cadherin downregulation. J Cell Sci 2004, I I7:1675-1685.

20. Boireau S, Buchert M, Samuel MS, Pannequin J, Ryan JL, Choquet A, Chapuis H, Rebillard X, Avances C, Ernst M, Joubert D, Mottet N, Hollande F: DNA-methylation-dependent alterations of claudin-4 expression in human bladder carcinoma. Carcinogenesis 2007, 28:246-258.

21. Kominsky SL, Argani P, Korz D, Evron E, Raman V, Garrett E, Rein A, Sauter G, Kallioniemi OP, Sukumar S: Loss of the tight junction protein claudin-7 correlates with histological grade in both ductal carcinoma in situ and invasive ductal carcinoma of the breast. Oncogene 2003, 22:202I-2033.

\section{Pre-publication history}

The pre-publication history for this paper can be accessed here:

http://www.biomedcentral.com/1471-2407/7/196/pre pub

\section{Publish with Biomed Central and every scientist can read your work free of charge}

"BioMed Central will be the most significant development for disseminating the results of biomedical research in our lifetime. "

Sir Paul Nurse, Cancer Research UK

Your research papers will be:

- available free of charge to the entire biomedical community

- peer reviewed and published immediately upon acceptance

- cited in PubMed and archived on PubMed Central

- yours - you keep the copyright
BioMedcentral 